\title{
Tp-e Interval and Tp-e/ QTc Ratio Are Significantly Increased in Patients with Brain Death
}

\author{
Abdullah Orhan Demirtaş*, [MD] \\ ORCID:0000-0003-4768-0536 \\ Örsan Deniz Urgun ${ }^{1},[\mathrm{MD}]$ \\ ORCID: 0000-0002-9125-4732 \\ Hasan Koca ', [MD] \\ ORCID:0000-0002-6232-4567 \\ Onur Kaypaklı ${ }^{2}$, [MD] \\ ORCID:0000-0002-5065-3283 \\ Yahya Kemal İçen $1,[\mathrm{MD}]$ \\ ORCID: 0000-0003-0070-5281 \\ Mevlüt Koç ${ }^{1}$ [MD] \\ ORCID:0000-0002-3000-4200 \\ 1 Department of Cardiology, University of Health \\ Sciences - Adana Health Practice and Research Center \\ Adana, Turkey \\ ${ }^{2}$ Department of Cardiology, Mustafa Kemal University \\ Faculty of Medicine, Hatay, Turkey \\ Corresponding Author: Abdullah Orhan Demirtaş, \\ Department of Cardiology, University of Health \\ Sciences - Adana Health Practice and Research Center, \\ Dr. Mithat Özsan Bulvarı Kışla Mah. 4522 Sok. No: 1 \\ Yüreğir, Adana, Turkey \\ e-mail: aorhandemirtas@gmail.com GSM: +90 \\ 5333109448
}

\section{reo ABSTRACT Clew}

Objective: We aimed to investigate whether there is a change in Tp-e interval, Tp-e/ QT and Tp-e/QTc ratios in patients with brain death.

Materials and Methods: Fifty brain death patients and 50 age and sex matched healthy controls were included in the study. In addition to routine evaluation, Tp-e interval, Tp-e / QT and Tp-e / QTc ratios were measured in 12-lead electrocardiography. Tp-e was measured in the precordial leads using the Tail method; the time from the peak of the $\mathrm{T}$ wave to the point where the wave reached the isoelectric line.

Results: White blood cell, aspartate aminotransferase, alanine aminotransferase and high sensitive $\mathrm{c}$ reactive protein levels were significantly higher in patients with brain death. The frequency of patients with QTc prolongation, Tp-e interval, Tp-e/QT and Tp-e/QTc values were significantly higher in patients with brain death $(\mathrm{p}<0.05)$. Tp-e interval, Tp-e/QT and Tp-e/QTc values were found to be positively correlated with HsCRP ( $<<0.001$ for all).

Conclusion: Tp-e interval, Tp-e / QT and Tp-e / QTc ratios are increased in patients with brain death compared to healthy controls. The mechanisms of this association and possible relationship with neuroendocrine changes should be investigated in future studies.

Keywords: Tp-e interval, Tp-e/QTc ratio, Brain death, QT interval

Received: 10 Agust 2019, Accepted: 11 September 2019,

Published online: 30 September 2019

\section{INTRODUCTION}

People diagnosed with brain death progress to somatic death in a few days due to vasodilatation and cardiac dysfunction due to sympathetic nervous system collapse caused by brain ischemia [1]. Pulmonary edema and diabetes insipidus in the early stages of brain death may accelerate this cardiopulmonary failure [2]. However, there is limited information about changes in electrocardiography (ECG) and cardiac arrhythmias in patients with brain death.

There are many ECG parameters related to ventricular repolarization and are associated with risk of ventricular arrhythmias. These parameters are QT and QTc time, QT and QTc dispersion and T wave peak to 
end interval (Tp-e interval). The Tp-e / QTc and Tp-e / QTc ratios are associated with ventricular transmural dispersion during repolarization [3]. Increased Tp-e interval shows abnormal spread of ventricular repolarization wave and is associated with increased risk of ventricular arrhythmias [4]. Brain death and acute brain injury were thought to affect the autonomic nervous system and their effects on ventricular repolarization, and thus Tp-e interval [5]. To the best of our knowledge, there are limited data on QT and QTc interval, QT and QTc dispersion for the risk of cardiac arrhythmias and the effect of brain death on ventricular repolarization $[5,6]$. However, there is no study on the Tp-e interval, Tp-e / QT and Tp-e / QTc ratios.

Therefore, in our study, we aimed to investigate whether there was a change in Tp-e interval, Tp-e / QT and Tp-e / QTc ratios in patients with brain death compared to the control group of the same age and gender.

\section{MATERIAL and METHODS}

\section{Patients Populations}

Between 2011 and 2019, 100 patients with a diagnosis of brain death were retrospectively reviewed in Adana Numune Training and Research Hospital and Adana City Hospital. Brain death diagnosis was made by a group of four medical doctors from anesthesia and reanimation, neurology, neurosurgery and cardiology as described in the 2014 Turkish Neurological Society's Brain Death Guideline [7]. 50 patients with brain death ( 29 males, 21 females and mean age $44.7 \pm 17.4$ years) and 50 healthy controls ( 27 males, 23 females and mean age 45.9 \pm 16.4 years) were included in the study. We selected age and sex matched healthy control group patients with no history of any medical condition from the outpatient unit of our cardiology clinic. From patients who were screened for brain death; patients under 18 years old, patients with suboptimal Tp-e and QTc measurements on the ECG examination, known coronary artery disease (CAD) or hypertension, diabetes mellitus from major risk factors of $C A D$, intermediate or advanced valvular heart disease, systolic heart failure, syncope or sudden cardiac arrest in the family or personal history, acute or chronic systemic or local infection and patients who use drugs known to prolong or shorten QT interval were excluded from the study. The study was conducted in accordance with the principles of Helsinki; and approved by the Local Ethics Committee (Adana City Hospital, Ethics Committee, 2018).

Laboratory values and echocardiography of all patients were examined. Demographic data of all patients; sex, height, weight, body mass index, blood pressure values were recorded from the files in the archive. Complete blood count, renal function tests, liver function tests, high sensitive $C$ reactive protein (hs-CRP) values were recorded from routine biochemistry parameters. From the recorded echocardiography images and reports; the patients were evaluated for organic heart disease, valvular heart disease or systolic dysfunction [8].

\section{2-lead Electrocardiographic Evaluation}

Twelve-lead ECG were registered on MAC 2000 ECG recorders (GE medical systems information technologies, Inc.,WI, USA) using a $25 \mathrm{~mm} / \mathrm{s}$ paper speed and $1 \mathrm{mv} / 10 \mathrm{~mm}$ standard calibration in patients with sinus rhythm. The time from the beginning of the $P$ wave to the beginning of the QRS complex was measured as the PR interval. The QT interval was calculated from beginning of the QRS complex to the end of the T wave. QTc was calculated

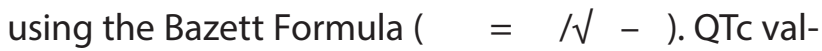
ues longer than $\mathbf{4 6 0}$ miliseconds in women and 450 miliseconds in men was accepted as prolonged QTC. Tp-e was measured in the precordial leads using the Tail method; the time from the peak or nadir of the $T$ wave to the point where the wave reached the isoelectric line [9]. Tp-e measurements were made primarily from V5 (Figure 1). If V5 is not suitable for measurement (amplitude $<1.5 \mathrm{~mm}$ ), measurements are made from V4 or V6 [10]. All ECG examinations were evaluated by two electrophysiologists who had at least 5 years of experience in electrophysiology and who were blind to the patient data. To decrease the error of the measurements, QT and Tp-e intervals were measured manually with calipers and magnifying glass. As a result of this evaluation, if there was an incompatible result between the two electrophysiologists (interobserver difference), the final decision was given by the 3rd electrophysiology specialist. 

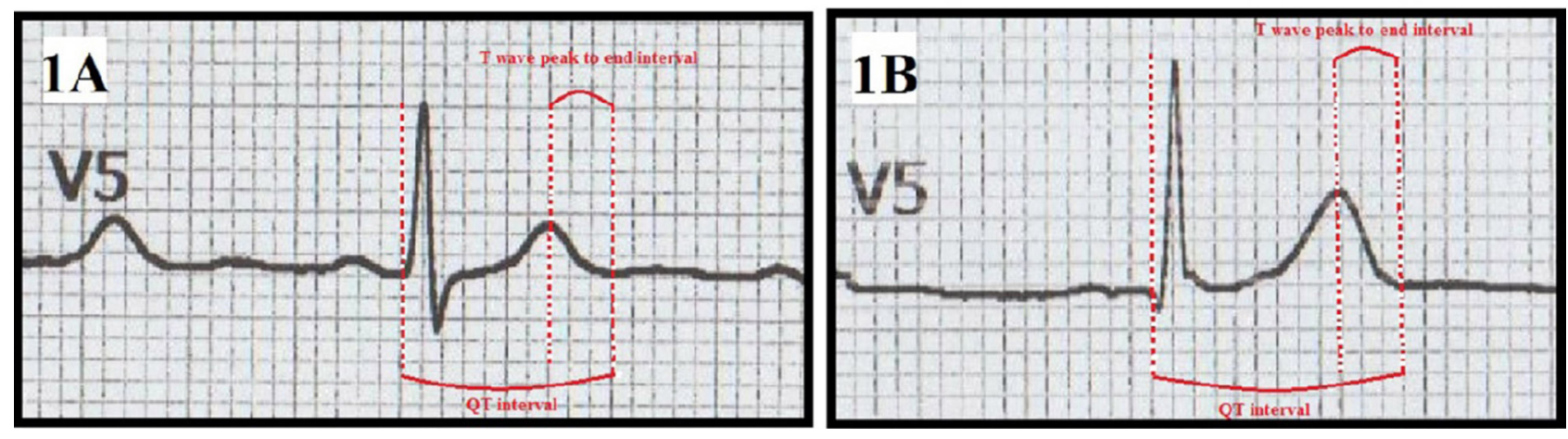

Figure 1. (A) Tp-e / QTc measurement in a patient with brain death, (B) Tp-e / QTc measurement in a patient without brain death

\section{Statistical Analysis}

Statistical analyses were performed using SPSS statistical software (version 20.0; SPSS Inc., Chicago, IL, USA). A two-sided $P$ value $<0.05$ was considered significant and the $\mathrm{Cl}$ was $95 \%$. Continuous variables were expressed as mean \pm SD or median (interquartile range) and categorical variables were expressed as percentages. Continuous variables were tested for normal distribution by the Kolmogorov-Smirnov test. Categorical variables were analyzed by chisquare test or Fisher's exact test. Comparisons of continuous variables between the two groups were performed using the Student's t-test. The kappa coefficient was used to examine the interobserver variability of the all ECG measurements. Pearson's and Spearman's correlation were used to examine the relationship between continuous variables.

\section{RESULTS}

Cohen kappa values that evaluate interobserver and intraobserver variability were over $90 \%$ for all ECG criteria.

Comparison of Demographic, Clinical and Laboratory Variables

Age and sex were found to be similar between groups. The blood pressure of the two groups was also similar. White blood cell (WBC), aspartate aminotransferase (AST), alanine aminotransferase (AST) and hsCRP levels were significantly higher in patients with brain death. Other laboratory data were similar between the two groups (Table 1).

Table 1. Comparison of demographic and laboratory findings

\begin{tabular}{|c|c|c|c|}
\hline & $\begin{array}{l}\text { Brain death group } \\
\qquad n=50\end{array}$ & $\begin{array}{c}\text { Control group } \\
n=50\end{array}$ & $\mathrm{p}$ \\
\hline Age (years) & $44.7 \pm 17.4$ & $50.9 \pm 16.4$ & 0.069 \\
\hline Male gender n (\%) & $29(48)$ & $27(54)$ & 0.859 \\
\hline $\begin{array}{l}\text { Systolic blood pressure, } \\
(\mathrm{mmHg})\end{array}$ & $132.9 \pm 13.4$ & $130.2 \pm 15.6$ & 0.354 \\
\hline $\begin{array}{l}\text { Diastolic blood pressure } \\
(\mathrm{mmHg})\end{array}$ & $74.1 \pm 10.9$ & $76.0 \pm 11.5$ & 0.397 \\
\hline Height $(\mathrm{cm})$ & $76.1 \pm 8.2$ & $165 . \pm 10$ & 0.301 \\
\hline Weight (kg) & $82.5 \pm 7.5$ & $73.5 \pm 6.3$ & 0.068 \\
\hline Body mass index $\left(\mathrm{kg} / \mathrm{m}^{2}\right)$ & $27.5 \pm 2$ & $27.2 \pm 1.7$ & 0.323 \\
\hline White blood cell (103/uL) & $12.8 \pm 6.5$ & $9.7 \pm 2.6$ & 0.002 \\
\hline Hemoglobin (g/dl) & $10.8 \pm 1$ & $11.2 \pm 1.1$ & 0.082 \\
\hline Platelet $(103 / \mu \mathrm{L})$ & $181.9 \pm 122.2$ & $121.2 \pm 86.5$ & 0.160 \\
\hline Urea (mg/dl) & $60.0 \pm 45.1$ & $31 \pm 8.5$ & 0.236 \\
\hline Creatinine (mg/dl) & $0.81 \pm 1.14$ & $0.74 \pm 0.13$ & 0.624 \\
\hline Glomerular filtration rate & 84.3 & 84.7 & 0.933 \\
\hline Sodium (mEq/L) & $143.6 \pm 11.2$ & $142.3 \pm 6.8$ & 0.490 \\
\hline
\end{tabular}




\begin{tabular}{|l|c|c|c|}
\hline Potassium (mEq/L) & $4.5 \pm 0.6$ & $4.31 \pm 0.5$ & 0.401 \\
AST (mg/dl) & $90.3 \pm 76$ & $30.0 \pm 13.7$ & $<0.001$ \\
ALT (mg/dl) & $50.0 \pm 23$ & $23.0 \pm 10.5$ & $<0.001$ \\
Hs-CRP & $59.7 \pm 0.3$ & $11.5 \pm 0.5$ & $<0.001$ \\
\hline
\end{tabular}

ALT: Alanine aminotransferase, AST: Aspartate aminotransferase CRP: C reactive protein

\section{Comparison of Electrocardiographic Variables}

When the electrocardiographic parameters were examined; The frequency of patients with QTc prolongation, Tp-e interval, Tp-e / QT and Tp-e / QTc values were significantly higher in patients with brain death

Table 2. Comparison of ECG findings

\begin{tabular}{|c|c|c|c|}
\hline & $\begin{array}{c}\text { Brain death group } \\
n=50\end{array}$ & $\begin{array}{c}\text { Control group } \\
n=50\end{array}$ & $\mathrm{p}$ \\
\hline Heart rate (bpm) & $80.4 \pm 16.2$ & $80.4 \pm 17.8$ & 0.995 \\
\hline PR interval (ms) & $146.0 \pm 17.9$ & $141.1 \pm 11.1$ & 0.108 \\
\hline QRS duration (ms) & $88.6 \pm 11.7$ & $87.5 \pm 10.8$ & 0.682 \\
\hline QRS axis & $38.5 \pm 36.6$ & $34.8 \pm 31.8$ & 0.594 \\
\hline Ventricular extra systole, n (\%) & $4(8 \%)$ & $0(0)$ & 0.117 \\
\hline $\begin{array}{l}\text { Supraventricular extra systo- } \\
\text { le, n (\%) }\end{array}$ & $4(8 \%)$ & $0(0)$ & 0.117 \\
\hline QT interval (ms) & $386.4 \pm 43.8$ & $372.4 \pm 45.6$ & 0.164 \\
\hline QTc interval (ms) & $422.1 \pm 63.4$ & $402.2 \pm 32.9$ & 0.093 \\
\hline $\begin{array}{l}\text { Patients with QTc prolonga- } \\
\text { tion, } \mathrm{n}(\%)\end{array}$ & $18(36 \%)$ & $0(\% 0)$ & $<0.001$ \\
\hline Tp-e interval (msn) & $92.3 \pm 18.9$ & $70.4 \pm 18.5$ & $<0.001$ \\
\hline Tp-e/QT & $0.24 \pm 0.1$ & $0.19 \pm 0$ & $<0.001$ \\
\hline Tp-e/QTc & $0.22 \pm 0.1$ & $0.18 \pm 0$ & $<0.001$ \\
\hline
\end{tabular}

QTc: Corrected QT interval

Correlation Analysis of ECG and Laboratory Parameters

Correlation analysis of ECG and laboratory parameters was shown in Table 3. Tp-e interval was found to be positively correlated with HsCRP ( $r=0.387$, $p<0.001)$, ALT $(r=0.251, p=0.012)$, AST $(r=0.249$, $p=0.012)$ and potassium values $(r=0.209, p=0.037)$ in bivariate analysis. Scatter plot diagram of the relationship of Tp-e interval with HsCRP is shown in

Table 3. Correlation analysis of ECG and laboratory data
Figure 2. Tp-e/QT ratio was found to be positively correlated with HsCRP $(r=0.348, p<0.001), A L T$ $(r=0.283, p=0.004)$, AST $(r=0.312, p=0.002)$ and WBC $(r=0.205, p=0.041)$ in bivariate analysis. Tp-e/QTc ratio was found to be positively correlated with HsCRP $(r=0.371, p<0.001)$, ALT $(r=0.275, p=0.006)$ and AST $(r=0.327, p=0.001)$ in bivariate analysis. Scatter plot diagram of the relationship of Tp-e/QTc ratio with HsCRP is shown in Figure 3.

\begin{tabular}{|l|c|c|c|c|c|c|}
\hline \multirow{2}{*}{} & \multicolumn{2}{|c|}{ Tp-e interval } & \multicolumn{2}{c|}{ Tp-e/QT ratio } & \multicolumn{2}{c|}{ Tp-e/QTc ratio } \\
Hs-CRP & $\mathrm{r}$ & $\mathrm{p}$ & $\mathrm{r}$ & $\mathrm{p}$ & $\mathrm{r}$ & $\mathrm{p}$ \\
ALT & 0.387 & $<0.001$ & 0.348 & $<0.001$ & 0.371 & $<0.001$ \\
AST & 0.251 & 0.012 & 0.283 & 0.004 & 0.275 & 0.006 \\
WBC & 0.249 & 0.012 & 0.312 & 0.002 & 0.327 & 0.001 \\
\hline
\end{tabular}

Tp-e: T wave peak to end interval, QTc: Corrected QT interval 


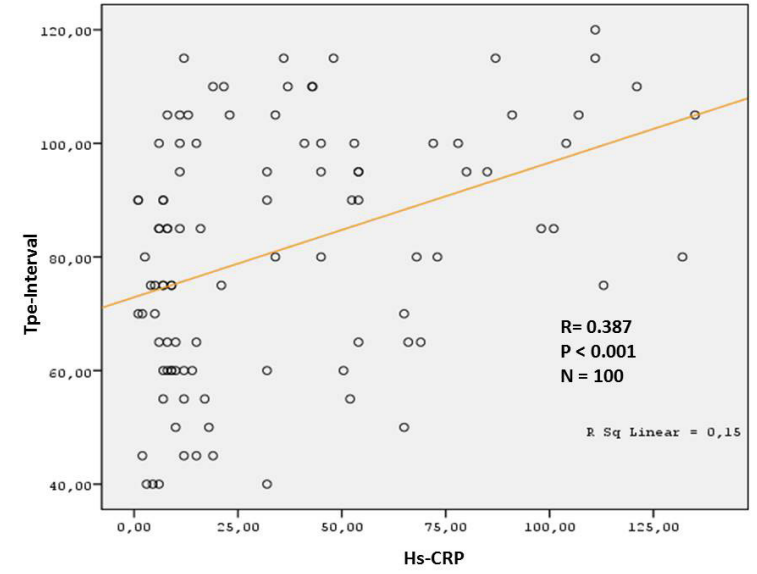

Figure 2. Scatter plot diagram of the relationship of Tp-e interval with HsCRP.

\section{DISCUSSION}

The main findings of the present study are that: 1) We found significant increase of Tp-e interval, Tp-e / QT and Tp-e / QTc ratios in patients with brain death compared to healthy controls. 2) Tp-e interval, Tp-e / QT and Tp-e / QTc ratios were found to be positively correlated with HsCRP. To the best of our knowledge, this is the first study to demonstrate significant increase of Tp-e interval, Tp-e / QT and Tp-e / QTc ratios from ventricular repolarization parameters in patients with brain death.

Depolarization of ventricular myocardium occurs from the endocardial region to the epicardial region. Ventricular repolarization follows ventricular depolarization. There is a dispersion between the endocardial and epicardial region during ventricular repolarization. The interval between the peak of the T wave and the end of the T wave is called the Tp-e interval and is associated with this transmural ventricular repolarization $[3,11]$. The Tp-e interval and the ratio of this interval to the QT interval have been shown to be associated with the arrhythmic events in many cardiac pathological conditions and also pose a high risk for sudden cardiac death [4,12-14]. The association of increased Tp-e interval and Tp-e / QT ratio with arrhythmia and SCD may be the result of the dispersion occurring in the repolarization between the epicardial and endocardial region of the ventricular myocardium which causes slow transmission and re-entry.

Although there are studies evaluating QT and QTC interval and QT dispersion from ventricular repolarization parameters in patients with brain death, there are no studies investigating Tp-e interval, Tp-e

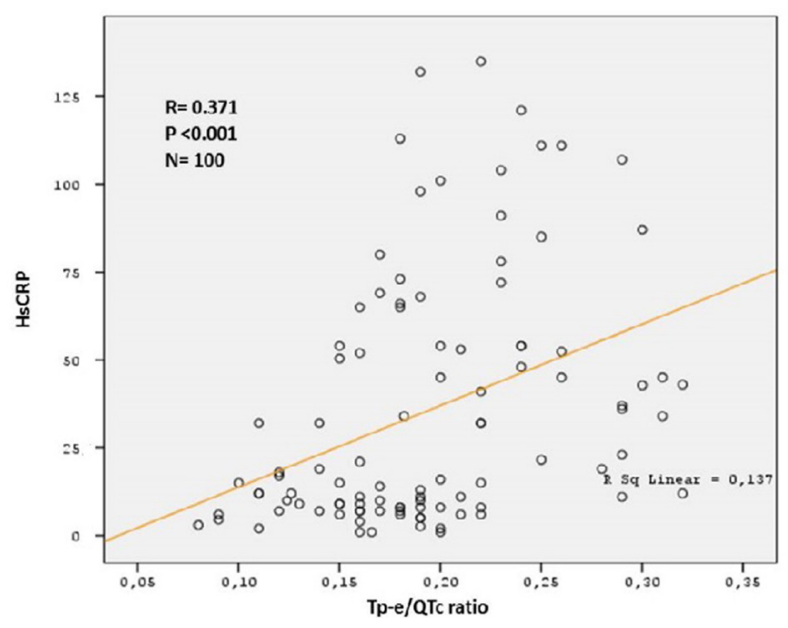

Figure 3. Scatter plot diagram of the relationship of Tp-e/QTc ratio with $\mathrm{HsCRP}$

/ QT and Tp-e / QTc ratio [5,6]. In a study by Vakilian et al. [5], heart rate variability was found to be lower in patients with brain death compared to patients with comatose state, but no significant difference was found between the two groups in terms of QT dispersion. In a study evaluating QTc interval in ECG in children with brain death by Plymale et al. [6] found that QTc duration was significantly higher in patients with brain death than healthy control group. In the same study, it was reported that increased QTc and low potassium and high calcium levels and male sex were positively related.

Several hypotheses that may be associated with QT prolongation in brain damage and brain death in adults have been considered. Reduced heart rate variability and reduction in baro-sensitivity caused by brain death and acute brain injury were thought to affect the autonomic nervous system and their effects on ventricular repolarization, and thus QT and QTc intervals $[5,6,15]$. Severity of brain damage has been shown to be associated with the severity autonomic and cardiovascular system changes $[5,15]$. Another possible cause of QT prolongation is increased activity in the sympathetic nervous system caused by brain damage [16].

The most common etiology of brain death in adults are trauma and subarachnoid hemorrhage, but there are some other causes with large-scale brain damage such as intracerebral hemorrhage, hypoxic ischemic encephalopathy, ischemic stroke $[17,18]$. In patients with subarachnoid hemorrhage, which is the most common cause of brain death, cardiac findings such as elevated troponins, NT-proBNP, 
cardiomyopathy with takotsubo-like apical hypokinesia, QT interval prolongation, T-wave inversion, ST-segment depression have been demonstrated in previous studies and evaluated as a sign of poor prognosis $[19,20]$.

Increased values of ventricular repolarization parameters such as QT and QTc duration, QTc dispersion, Tp-e interval, Tp-e / QTc have shown to be risk factors for ventricular arrhythmias and death $[3,4,21]$. In the literature, QT and QTc duration and QTc dispersion have been shown to be increased in patients with brain death in a limited number of studies $[5,6]$. Nevertheless, there are no studies regarding the change of Tp-e interval, Tp-e/QT and Tp-e/QTc. Our study is the first to demonstrate significant increase in Tp-e interval, Tp-e/QT and Tp-e/QTc ratios in patients with brain death. In our study, although the frequency of patients with long QT was more frequent in brain death group, mean QT and QTc durations were found to be similar in brain death and healthy control groups. However, the Tp-e interval, Tp-e / QT and Tp-e / QTc ratio were longer in patients with brain death. This finding may be related to the characteristics of our patient population and the fact that some conditions causing QT prolongation were excluded.

There are some important limitations in our study. One of them is the retrospective design of the study and the number of patients included in the study.
Because of the retrospective nature of our study, we were not able to specify the relationship between the causes of brain death and Tp-e values. In addition, due to the small number of patients and lack of clinical follow-up, association with the clinical arrhythmias and other clinical follow-up parameters were not evaluated. Prospective and more comprehensive studies can provide further information. In our study, patients using medical treatment which causes QT prolongation were excluded; however, no genetic evaluation was made for long or short QT syndromes. These inherited channelopathies have been thought to have minor effect on our study, because of their low prevalence. As a result of the nature of our study, the results do not reveal a causal relationship, but only demonstrate an association. In Conclusion, Tp-e interval, Tp-e / QT and Tp-e / QTc ratios are increased in patients with brain death compared to healthy controls. For patients with brain death, especially for organ donors; It should be kept in mind that prolongation of ventricular repolarization may occur. The mechanisms of this association and possible relationship with neuroendocrine changes should be investigated in future studies.

\section{CONFUCT OF INTEREST}

We have not any conflict of interest in this study. 


\section{re) REFERENCES Cem}

[1] Wood KE, Becker BN, McCartney JG, et al. Care of the potential organ donor. N Engl J Med 2004; 351:2730

[2] Wijdicks EF. Determining brain death in adults. Neurology 1995; 45:1003.

[3] Kongstad O, Xia Y, Liang Y, et al. Epicardial and endocardial dispersion of ventricular repolarization. A study of monophasic action potential mapping in healthy pigs. Scandinavian Cardiovascular Journal 2005; 39: 342-47.

[4] Porthan K, Viitasalo $M$, Toivonen $L$, et al. Predictive Value of Electrocardiographic T Wave Morphology Parameters and T-Wave Peak to T-Wave End Interval for Sudden Cardiac Death in the General Population Clinical Perspective. Circulation: Arrhythmia and Electrophysiology 2013; 6: 690-96.

[5] Vakilian AR, Iranmanesh $F$, Nadimi $A E$, et al. Heart rate variability and QT dispersion study in brain death patients and comatose patients with normal brainstem function. J Coll Physicians Surg Pak 2011; 21: $130-33$

[6] Plymale J, Park J, Natale J, et al. Corrected QT Interval in Children With Brain Death. Pediatr Cardiol 2010; 31: 1064-69.

[7] Arsava EM, Demirkaya Ş, Dora B, et al. Turkish Neurological Society Diagnostic Guidelines for Brain Death Turk J Neurol 2014; 20: 101-04.

[8] Lang RM, Bierig M, Devereux RB, et al. Recommendations for chamber quantification. European journal of echocardiography. 2006; 7:79-108

[9] Salles GF, Cardoso CR, Leocadio SM, et al. Recent ventricular repolarization markers in resistant hypertension: Are they different from the traditional QT interval? Am J Hypertens 2008; 21: 47- 53.

[10] Chua KC, Rusinaru C, Reinier K, et al. T peak-to-Tend interval corrected for heart rate: $A$ more precise measure of increased sudden death risk? Heart rhythm 2016; 13: 2181-85

[11] Xia Y, Liang Y, Kongstad O, et al. T peak-T end interval as an index of global dispersion of ventricular repolarization: evaluations using monophasic action potential mapping of the epi- and endocardium in swine. J Interv Card Electrophysiol 2005; 14: 79-87.

[12] Gupta P, Patel C, Patel H, et al. T(p-e)/QT ratio as an index of arrhythmogenesis. J Electrocardiol 2008; 41: 567-74.

[13] Erikssen G, Liestol K, Gullestad L, et al. The terminal part of the QT interval ( $T$ peak to $T$ end): a predictor of mortality after acute myocardial infarction. Ann Noninvasive Electrocardiol 2012; 17: 85-94.

[14] Smetana P, Schmidt A, Zabel M, et al. Assessment of repolarization heterogeneity for prediction of mortality in cardiovascular disease: peak to the end of the $T$ wave interval and nondipolar repolarization components. J Electrocardiol 2011: 44: 301-308.

[15] Goldstein B, Toweill D, Lai S, et al. Uncoupling of the autonomic and cardiovascular systems in acute brain injury. Am J Physiol 1998; 275: R1287- R92.

[16] Cheung RT, Hachinski V. The insula and cerebrogenic sudden death. Arch Neurol 200; 57: 1685- 88.

[17] Saposnik G, Bueri JA, Mauriño J, et al. Spontaneous and reflex movements in brain death. Neurology 2000; 54: 221-23.

[18] Goudreau JL, Wijdicks EF, Emery SF. Complications during apnea testing in the determination of brain death: predisposing factors. Neurology 2000; 55: 1045-48.

[19] Lee M, Oh JH, Lee KB, Kang GH, et al. Clinical and Echocardiographic Characteristics of Acute Cardiac Dysfunction Associated With Acute Brain Hemorrhage区- Difference From Takotsubo Cardiomyopathy. Circ J 2016 Aug 25; 80: 2026-32.

[20] Frontera JA, Parra A, Shimbo D, et al. Cardiac Arrhythmias after Subarachnoid Hemorrhage: Risk Factors and Impact on Outcome. Cerebrovasc Dis 2008; 26: 71-78.

[21] Gölbaşi Z, Selçoki Y, Eraslan T, et al. QT dispersion. Is it an independent risk factor for in-hospital mortality in patients with intracerebral hemorrhage? Jpn Heart J 1999; 40: 405-11. 